\title{
NOTES AND DISCUSSION
}

\section{Processing of Lexical Ambiguities: A Comment on Milberg, Blumstein, and Dworetzky (1987)}

\author{
Peter Hagoort \\ Max-Planck-Institute for Psycholinguistics, Nijmegen
}

\begin{abstract}
In a study by Milberg, Blumstein. and Dworetzky (1987), normal control subjects and Wernicke's and Broca's aphasics performed a lexical decision task on the third element of auditorily presented triplets of words with either a word or a nonword as target. In three of the four types of word triplets, the first and the third words were related to one or both meanings of the second word, which was semantically ambiguous. The fourth type of word triplet consisted of three unrelated, unambiguous words, functioning as baseline. Milherg et al. (1987) claim that the results for their control subjects are similar to those reported by Schvaneveldt, Meyer, and Becker's original study (1976) with the same prime types, and so interpret these as evidence for a selective lexical access of the different meanings of ambiguous words. It is argued here that Milberg et al. only partially replicate the Schvaneveldt et al. results. Moreover, the results of Milberg et al. are not fully in line with the selective access hypothesis adopted. Replication of the Milberg et al. (1987) study with Dutch materials, using both a design without and a design with repetition of the same target words for the same subjects led to the original pattern as reported by Schvaneveldt et al. (1976). In the design with four separate presentations of the same target word, a strong repetition effect was found. It is therefore argued that the discrepancy between the Milberg et al. results on the one hand, and the Schvaneveldt et al. results on the other, might be due to the absence of a control for repetition effects in the within-subject design used by Milberg et al. It is concluded that this makes the results for both normal and aphasic subjects in the latter study difficult to interpret in terms of a selective access model for normal processing. (c) 1989 Academic Press, Inc.
\end{abstract}

This research was supported by a stipend from the Max-Planck-Gesellschaft zur Förderung der Wissenschaften. I thank Di Bradley, Colin Brown, Pim Levelt, Rob Schreuder, and Pienie Zwitserlood for their helpful comments on earlier versions of this paper, and Niels van Haarlem for running the subjects. Address correspondence and reprint requests to Peter Hagoort, Max-Planck-Institute for Psycholinguistics, Wundtlaan 1, 6525 XD Nijmegen, The Netherlands. 


\section{INTRODUCTION}

In a number of recent studies using a semantic priming paradigm with a lexical decision task, it has been demonstrated that Wernicke patients produce essentially the same pattern of results as normal control subjects, whereas Broca patients seem to be less or not at all sensitive to primetarget relations (Milberg \& Blumstein, 1981; Blumstein, Milberg, \& Shrier, 1982; Milberg et al., 1987). This differential outcome is most clear in the recent Milberg et al. study (1987), which is based both in design and in materials on Schvaneveldt et al.'s work (1976) on the processing of lexical ambiguity in normal subjects. The Milberg et al. findings are intriguing, not in the least because of their clear discrepancy with the standard view that lexical-semantic processing is especially disturbed in Wernicke patients, whereas it is not or is less disturbed in Broca patients (see Caramazza \& Berndt, 1978).

The logic underlying most modern psycholinguistically oriented work in aphasiology is that conclusions as to the functional deficits of aphasic patients are to be based upon a comparison of their performance with that of normal speakers, in experimental situations which reveal significant aspects of language processing. That is, the data for the normal group are to serve as a baseline, in the light of which the pattern of results for the aphasic patients is to be interpreted. What is presupposed is that one can account for the normal data in terms of well-established psycholinguistic models.

Following this two-step reasoning, Milberg et al. (1987) first report that their results for normal subjects are "similar to those reported by Schvaneveldt, Meyer, and Becker (1976)" (pp. 144-145). In agreement with the latter authors, the normal subject data are interpreted as evidence in favor of a "selective access" model for the processing of ambiguous words. That is, of the separate meanings of a lexical ambiguity, only the one that fits the prior semantic context is said to be accessed. The results for the groups of Wernicke's and Broca's aphasics are subsequently interpreted with reference to the normal processing model: Wernicke patients "show, as do normals, selective access to different meanings of ambiguous words," whereas "the lack of semantic facilitation in any of the priming conditions for the Broca's aphasics supports the intriguing possibility that these patients have a deficit in the processing of semantic information" (p. 147). In this note, the first step in the two-step argument is addressed, in that the "similarity" with the Schvaneveldt et al. data will be questioned.

Milberg et al. used four of the six prime conditions and a subset of the triplet materials from the Schvaneveldt et al. (1976) study, where the final elements were either words or pronounceable nonwords. In the critical word target set, the triplets consisted of two primes and a target. 
In three of the four prime conditions the second prime was an ambiguous word. In the concordant condition, the first prime and the target were related to each other and to just one meaning of the second, ambiguous prime (e.g., coin-bank-MONEY). In the discordant condition, the first prime was related to one meaning of the ambiguity, whereas the target was related to another (e.g., river-bank-MONEY). The neutral condition consisted of triplets in which the first prime was unrelated to the middle ambiguous prime, while the target was related (e.g., desk-bank-MONEY). The unrelated (baseline) condition consisted of three unrelated, unambiguous words (e.g., nurse-fish-MONEY).' The triplets were presented auditorily, and the subject's task was to make a lexical decision about the final target element.

With the semantic word contexts provided by these materials, what is being measured with a lexical decision task is the effect of intralexical priming, which is usually taken to be a function of the semantically based organization of the lexical network and of the automatic spread of activation to related elements (Seidenberg, Tanenhaus, Leiman, \& Bienkowski, 1982). This type of lexical context effect can be accounted for not only within interactive models of word recognition, but is also easily incorporated within a modular view on the processes of word recognition (Forster, 1979), and does not contradict the reported findings of multiple access in a nonlexical semantic context (Seidenberg et al., 1982; Swinney, 1979).

Within the tradition of intralexical priming, "selective access" is the activation of only one meaning of an ambiguous word within a word context. For the research under discussion, it means that activation from the first word in the triplet spreads only to the related meaning of the ambiguous word, which gains further activation with the presentation of the ambiguous word itself. In consequence, there is an automatic bias as to which reading of the ambiguous word is selected by the context. The extent to which activation is carried through to the target word depends upon the direction of the reading selection within the prime pair. In the discordant condition, no priming effect is expected because the meaning of the target word (e.g., MONEY) relates to a reading of its immediately preceding ambiguous prime other than the one selected by the initial prime (e.g., river-bank). The account here spells out Milberg et al.'s assumption that the discordant condition should gain no facilitation relative to the baseline, where two unambiguous primes are each unrelated to the target. In the neutral condition neither reading of the ambiguous word benefits from the activation spread from the unrelated first word.

'Milberg et al.'s construction of the unrelated triplets differed in one respect from those of Schvaneveldt et al. The latter authors had no triplets consisting solely of unambiguous words. Because one does not know the contribution of the ambiguity itself, it might have been better to also construct the unrelated triplets (the baseline) in the same way as Schvaneveldt et al. did: three unrelated words of which the second is ambiguous. 
TABLE 1

Mean Reaction Time in Lexical Decision as a Function of Priming Condition ${ }^{a}$

Milberg et al.

Schvaneveldt et al. (1976)

(1987)

\begin{tabular}{lcccccc} 
Priming condition & Expt. 1 & $d$ & Expt. 2 & $d$ & Expt. 1 & $d$ \\
\hline Concordant & 505 & 33 & 617 & 54 & 779 & 33 \\
Discordant & 558 & -20 & 662 & 9 & 792 & 20 \\
Neutral & 521 & 17 & 635 & 36 & 767 & 45 \\
Unrelated & 538 & & 671 & & 812 &
\end{tabular}

${ }^{a}$ Differences $(d)$ measured relative to the unrelated baseline. The data of Milberg et al. are reconstructed from their Figure 1 (p. 145).

Therefore, on presentation of the ambiguous prime itself, either both readings get some activation, or perhaps stochastically, the one related to the target is accessed in some instances, and in others the alternative unrelated meaning (Schvaneveldt et al., 1976). Whichever of these possibilities might be correct, they both predict more facilitation for the neutral condition than for the discordant condition. Thus Milberg et al. summarize by saying that if there is "more facilitation for the neutral condition than the discordant condition, and if the discordant condition produced no facilitation relative to the baseline condition, then it would suggest that . . access . . was selective (i.e., it was affected by context, namely, the semantic representation of the first prime word)" (p. 142).

However, this picture cannot be complete without a consideration of the concordant condition, so far omitted in this outline of a selective access hypothesis. To accord with that hypothesis, responses to targets in the concordant condition should be faster than in all other conditions, and certainly there should be significantly more facilitation than in the discordant condition. With concordant primes, activation from the first word not only spreads to the target-related reading of the ambiguous word, but also to the target itself (Schvaneveldt \& Meyer, 1973). On the basis of the logic that underlies predictions for the neutral, discordant, and unrelated conditions, one should expect the greatest facilitation for the concordant primes. And that is exactly what Schvaneveldt et al. found in the experiments they reported. Table 1 summarizes these data and compares them with the normal subject data of Milberg et al. (1987).

Although Milberg et al. report that their results are "similar" to those of Schvaneveldt et al. (1976), closer inspection reveals, critically, that priming in the concordant condition is neither greater than in the neutral condition nor securely different from the discordant one. The selective access hypothesis which is consistent with the Schvaneveldt et al. results is not fully consistent with the pattern in the Milberg et al. study, examined 
in this detail. In short, the claim of similarity is unwarranted, and the data of Milberg et al. for their normal subjects remain in need of a coherent story. And this is a matter for real concern, since a more precise account of the aphasic deficits in terms of the selective access model adopted for the unimpaired subjects is thereby excluded.

Is there a possible explanation for the discrepancy between the results of Milberg et al. on the one hand and those of Schvaneveldt et al. on the other? A first difference between both studies is a procedural one: the subjects of the Schvaneveldt et al. experiments responded to each element of the visually presented triplets, while Milberg et al.'s subjects responded only to the triplet-final target, under auditory presentation. A second contrast with the earlier study concerns the design: Milberg et al.'s subjects heard every target word in all four priming conditions. It is possible that this degree of intimacy with the experimental materials and, thereby, with the structure of the experiment, might introduce special strategies, so that, for example, subjects might attend to both meanings of the ambiguous word under attentional control. Further, the possibility cannot be ruled out that semantic priming effects were "polluted" by repetition effects, given that the same target word was presented four times to each subject (Scarborough, Cortese, \& Scarborough, 1977). Apparently, Milberg et al. did not explicitly control in their design for possible effects of repetition: their single, fixed presentation order is reported to be random, not pseudorandom as is required to control for possible repetition effects.

To find out whether one (or maybe both) of these latter factors played a role in the findings of Milberg et al., two replication studies with auditory presentation were run, one in which no subject heard a specific word more than once and one in which the same design was used as in the Milberg et al. study, but with a counterbalancing to control for possible repetition effects.

\section{EXPERIMENT 1}

Experiment 1 was intended to be a close replication of the Milberg et al. experiment, with three changes. First, the stimulus assignment counterbalanced words across subjects, so that no individual subject heard the same word more than once. Second, the test language was Dutch, rather than English. Third, type of ambiguity was introduced as a separate factor, since there is some experimental evidence that in a priming context there is selective access for Noun-Noun ambiguities, but multiple access for Noun-Verb ambiguities (Seidenberg et al., 1982). Thus, one-half of the ambiguous words in the experimental sets consisted of Noun-Noun ambiguities (e.g., bank; "bank/bank"), and the other half consisted of Noun-Verb ambiguities (e.g., varen; "fern/to sail"). 


\section{Method}

Subjects. Forty subjects from the subject pool of the Max-Planck-Institute participated in this experiment. All were native speakers of Dutch, and were paid for their participation.

Materials. The stimuli in this study consisted of triplets in which the first and second members served as primes and the third as target. In three of the four priming conditions for real word targets, the second prime was a word with two or more unrelated meanings. The stimulus words were taken from an extensive pretest of 190 ambiguous Dutch words, in which the relative frequencies of the distinct meanings as well as the first associates in the distinct semantic fields were established for each ambiguity. This pretest was done with 70 subjects, mainly students from the University of Nijmegen. From this initial set of materials, 16 Noun-Noun ambiguities, 15 Noun-Verb ambiguities, and 1 AdjectiveVerb ambiguity were selected, all with a relatively strong associate for both meanings (with a mean of $48 \%$ of agreement in responses given for each semantic field; see Appendix for the set of experimental word triplets). One associate was used as the triplet-final target. A second order associate for the ambiguous words was established via a second pretest, in which subjects were presented with a pair consisting of the ambiguous word preceded by the associate of one reading in one version of this pretest, and by the associate of the other reading in the other version. The subjects were instructed to write down a word to complete the triplet. By this procedure the set of first primes for the experimental word triplets was established.

As in the Milberg et al. (1987) study, there were four priming conditions for word targets. In the concordant condition, the first prime and the target were associated with the same meaning of the second (ambiguous) prime (e.g., bier-kater-DRANK; "beer-tomcat/hangoverdrink"). In the discordant condition, the first prime and the target word were associated with different meanings of the second prime (e.g., poes-kater-DRANK; "puss-tomcat/hangover-drink"). In the neutral condition, the first word was unrelated to the second, but the third word was related to one of the meanings of the second word (e.g., pianokater-DRANK; "piano-tomcat/hangover-drink"). Finally, in the unrelated condition, the three words were unrelated and unambiguous (e.g., poes-piano-DRANK; "puss-pianodrink"). In addition to the 32 word triplets, there were 32 triplets in which the target was a pronounceable nonword, and the primes were previously unused words. In half of these nonword triplets, the first 2 words were unrelated and unambiguous (e.g., hemd-ketelREFEL; "shirt-kettle-refel"); in the other half, the second word was ambiguous and the first was related to one of its meanings (e.g., ladder-sport-ZORWAAL; "ladder-rung/sportzorwaal").

The test stimuli were arranged in two blocks: one block of 16 Noun-Noun word and 16 Noun-Noun nonword triplets, and a secund block of 16 Noun-Verb word (including 1 Adjective-Verb triplet) and 16 Noun-Verb nonword triplets. Each block was preceded by 12 practice trials. In addition, every test session started with 20 "starter" triplets to acquaint the subjects with the procedure.

All materials were spoken by a female speaker in a sound-proof booth and recorded on a Revox A 700 tape recorder. The stimuli were digitized into a VAX 750 computer with a sampling rate of $20 \mathrm{kHz}$. Four test tapes were then constructed for the four versions of the experiment. In each version a test block consisted of four concordant, four discordant, four neutral, and four unrelated word triplets and a equal number of nonword triplets. The triplet assignment counterbalanced the words across subjects so that no individual subject heard the same item more than once. There was a 4-sec interval of silence between each triplet and a $0.5-\mathrm{sec}$ interval of silence between each item within a triplet. The input to and the output from the computer were low-pass filtered with a cutoff frequency of 10 $\mathrm{kHz}$.

Apparatus. The apparatus for the experiment consisted of a Revox B77 stereo tape recorder, a Miro GD laboratory computer, a pulse read unit, two pairs of headphones (one 
TABLE 2

Mean Auditory lexical Decision Times (RT) for Experiment 1 as a Function of Priming CONDITION $^{a}$

\begin{tabular}{llr}
\hline Priming condition & RT & $d$ \\
\hline Concordant & 621 & 75 \\
Discordant & 689 & 7 \\
Neutral & 667 & 29 \\
Unrelated & 696 &
\end{tabular}

${ }^{a}$ Differences $(d)$ measured relative to the unrclated baseline.

pair for the subject and another for the experimenter), and a response keyboard with a yes-button on the right side of the board and a no-button on the left. The test stimuli on the left channel of the tape were played binaurally to the subject, while pulses on the right channel of the tape at the onset of the targets, inaudible to the subjects, started a millisecond timer. The timer was stopped as soon as the subject pressed one of the buttons, and the data were automatically stored. The time-out was set to $2 \mathrm{sec}$.

Procedure. Subjects were assigned randomly to one of the four experimental versions (i.e., 10 subjects in each version). Each subject was tested individually in a single session lasting approximately $25 \mathrm{~min}$. Subjects were seated in a sound-proof booth with the keyboard placed in front of them, pressing the yes-button with the right index finger and the nobutton with the left.

Subjects were told that they would hear a series of triplets either ending with a word or ending with a nonword, and were instructed to respond to the third member of the triplet as quickly as possible, indicating whether it was a word by pushing the yes-button or a nonword by pushing the no-button. After the familiarization "starters," the subjects were asked to increase the speed of responding without losing accuracy. No further feedback was given during the testing session.

\section{Results}

The analyses that will be reported are on the reaction time data only, since the error rates were rather low: $2 \%$ for the words, and $3 \%$ for the nonwords. The effects of priming condition (four levels) and type of ambiguity (two levels) were tested in two analyses of variance, a subject analysis $\left(F_{1}\right)$ and an item analysis $\left(F_{2}\right)$. The results are summarized in Table 2 . There were significant main effects of priming condition $\left(F_{1}(3\right.$, $\left.117)=24.9, p<.001 ; F_{2}(3,90)=23.4, p<.001\right)$ and type of ambiguity $\left(F_{1}(1,39)=226.2, p<.001 ; F_{2}(1,30)=16.0, p<.001\right)$, but no significant interaction between these factors $\left(F_{1}\right.$ and $\left.F_{2}<1\right){ }^{2}$

${ }^{2}$ The effect for ambiguity type is not very revealing. It possibly is caused by factors that are not of immediate interest here, such as differences in the length and frequency of the target words within the types. The absence of a significant interaction with priming condition, however, is revealing. It shows that there is no special contribution of the wordclass difference to the effects of priming conditions. In contrast to Seidenberg et al. (1982), the present study finds no evidence for multiple access of Noun-Verb ambiguities in a priming context. 
Post hoc comparisons were carried out on the means of the four priming conditions using the Newman-Keuls procedure (with the relevant error term from the subject analysis and the item analysis, separately), and with the significance level set at .05 . These indicated in both cases that the concordant condition resulted in faster reaction times than all other conditions. Further, the neutral condition was significantly faster than both the discordant and the unrelated conditions, and there was no significant difference between the discordant condition and the unrelated baseline.

\section{Discussion}

These results replicate the original findings of Schvaneveldt et al., despite differences in modality of presentation (visual vs. auditory), experimental procedure (decisions on every word of the triplet vs. decisions on the final item of the triplet), and materials and language used. In contrast, despite the same modality of presentation and the same procedure as in the Milberg et al. study, this study fails to replicate their results in important respects. In accordance with the selective access hypothesis, but in contrast to the Milberg et al. results, a significant difference in the right direction between the concordant and the neutral condition was found. The most obvious possible cause of discrepancy is the design in the Milberg et al. study, either leading to strategy effects or to repetition effects for which no control was built in. Therefore, a second experiment was run with the same materials, but with a design in which all subjects heard all the experimental items in every condition in one and the same experimental session. However, in contrast to the Milberg et al. study, Experiment 2 controlled for possible effects of repetition.

\section{EXPERIMENT 2}

\section{Method}

Subjects. Twelve subjects from the subject pool of the Max-Planck-Institute participated in this experiment. They were paid for their participation.

Materials. The 128 word triplets were the same as in Experiment 1: 32 triplets in the concordant condition, 32 discordant, 32 neutral, and 32 unrelated. In half of the 64 nonword triplets an ambiguous prime was followed by a word related to one of its meanings; in the other half, the first and the second primes were unrelated and unambiguous. Every nonword triplet appeared twice in the experimental session. In this way, the materials were constructed in exactly the same way as in the Milberg et al. study. However, unlike the Milberg et al. procedure, the order of the four experimental word-target conditions was explicitly counterbalanced among triplets. This was done by taking two random samples of 16 from the 24 possible condition orders, one for the block of Noun-Noun items and one for the block of Noun-Verb items. These 16 orders were assigned randomly to the 16 basic word triplets per block. In this way, potential repetition effects due to presenting the same target word four times could be controlled.

The full experiment thus had 256 experimental triplets, presented in two blocks of 128 . 
TABLE 3

Mean auditory Lexical Decision Times (RT) for Expfriment 2 as a Function of Priming CONDITION $^{a}$

\begin{tabular}{llr}
\hline Priming condition & RT & $d$ \\
\hline Concordant & 596 & 52 \\
Discordant & 656 & -8 \\
Neutral & 622 & 26 \\
Unrelated & 648 & \\
\hline
\end{tabular}

${ }^{a}$ Differences $(d)$ measured relative to the unrelated baseline.

Every block was preceded by 12 practice items. The experimental session began with a set of 20 starter items to familiarize subjects with the task.

Procedure. Subjects were run individually, each session lasting approximately $45 \mathrm{~min}$, with the procedure as in Experiment 1. There was a short break between blocks 1 and 2 .

Results

Again, the error rates were low $(0.8 \%$ for the words, and $3.6 \%$ for the nonwords), so that analyses were carried out on reaction time data only. The results are summarized in Table 3 . Analyses of variance tested the effects of priming condition and type of ambiguity. There were significant main effects for priming condition $\left(F_{1}(3,33)=19.6, p<.001 ; F_{2}(3,90)\right.$ $=8.0, p<.001)$ and type of ambiguity $\left(F_{1}(1,11)=67.8, p<.001\right.$; $\left.F_{2}(1,30)=7.6, p<.001\right)$. There was, however, no significant interaction between priming condition and type of ambiguity $\left(F_{1}(3,33)=1.29, p\right.$ $\left.>.25 ; F_{2}<1\right)$.

Post hoc comparisons between the means of the four priming conditions were made, as in Experiment 1. A Newman-Keuls test using the error term from the subject analysis revealed significant differences $(p<.05)$ between all pairs of means, except--as in Experiment 1 -for the discordant versus the unrelated comparison. Using the error term from the item analysis, similar results were obtained with the exception that the difference between the concordant and neutral conditions failed to reach significance.

In addition to the effects for priming condition and type of ambiguity, the effect of repetition of target words was also tested, since every subject heard the same target word four times. In this analysis, all target words with the same index of presentation (first, second, and so on) were collapsed. Analyses of variance with index of presentation (four levels) as factor showed a highly significant effect for index of presentation $\left(F_{1}(3,33)=19.9, p<.001 ; F_{2}(3,93)=13.7, p<.001\right)$. The results are summarized in Table 4. Post hoc Newman-Keuls tests revealed significant differences $(p<.05)$ between the first presentation of the target words and all the following presentations. A separate analysis of variance 
TABLE 4

Mean Auditory Lexical Decision Times (RT) in Experiment 2 as a Function of the Index of PRESENTATION $^{a}$

\begin{tabular}{lll}
\hline Index of presentation & RT & $d$ \\
\hline First presentation & 678 & \\
Second presentation & 627 & 51 \\
Third presentation & 614 & 64 \\
Fourth presentation & 603 & 75 \\
\hline
\end{tabular}

${ }^{a}$ Difference $(d)$ relative to the first presentation.

on the means for all items with the same index of presentation within every priming condition showed a significant interaction between priming condition and index of presentation $\left(F_{1}(9,99)=2.4, p<.05\right)$. This interaction did not reach significance, however, when the degrees of freedom were adapted according to the Greenhouse and Geisser procedure (Winer, 1971) $\left(F_{1}(1,11)=2.4, p>.10\right)$.

\section{GENERAL DISCUSSION}

Both Experiment 1 and Experiment 2 replicated the results of the Schvaneveldt et al. study, but failed to replicate the results of the Milberg et al. study, despite the fact that Experiment 2, in particular, closely paralleled the latter. In fact, the only important difference in the design of their study and Experiment 2 was that I controlled explicitly for possible repetition effects, whereas they apparently did not. Not unexpectedly, the repetition effect is shown to be very strong when systematically examined. It seems therefore likely that the failure of the Milberg et al. study to find the same results as the Schvaneveldt et al. study and the experiments of this study may be attributable to the lack of an explicit control for repetition effects. ${ }^{3}$ It is therefore difficult to interpret the results for their normal control subjects with reference to the adopted psycholinguistic model (the selective access model).

This difficulty in interpreting the results for the normal control subjects does not invalidate the difference reported by Milberg et al. between the

\footnotetext{
${ }^{3}$ One of the reviewers suggested that the difference in results between the Milberg et al. study and this study might have to do with the fact that the nonaphasic control subjects in the former study were of an older age, resulting in a decrease in experimental sensitivity. This explanation, however, does not hold. In a follow-up of this study, in addition to a group of aphasic patients, a group of nonaphasic control subjects $(n=12)$ matched in age and education with the aphasic patients, was tested. This group, with a mean age of 58 years, showed an overall RT that was comparable to the overall RT of the group of control subjects in the Milberg et al. study. However, the pattern of results and the size of the priming effects were the same as in Experiment 2 of my study.
} 
Broca and the Wernicke patients. The normal pattern of performance for the Wernicke patients and the absence of any priming effect for the Broca patients certainly are relevant tindings, which are in need of further research and clarification. According to the logic of the two-step argument, however, the absence of data in support of the normal processing model adopted in the Milberg et al. study excludes at present a fine-grained interpretation of their patient data in terms of such a model. 


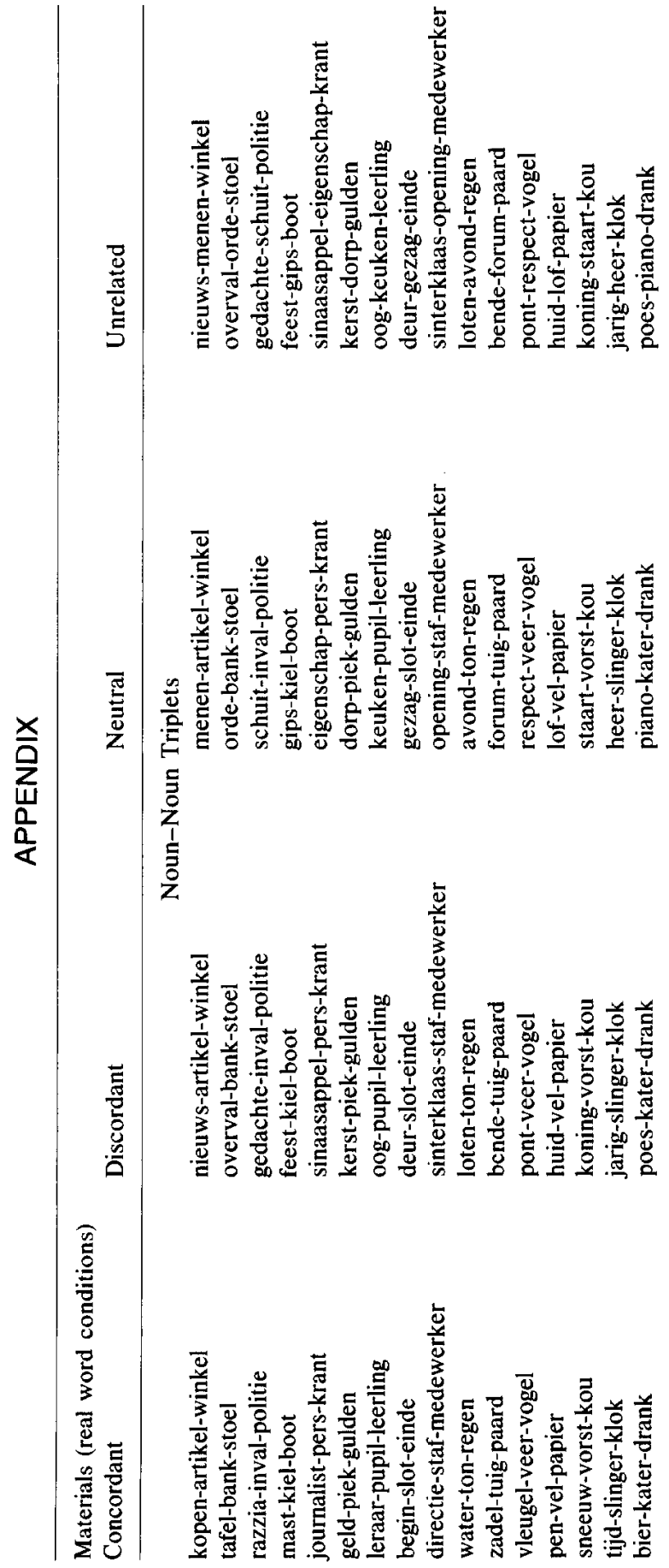



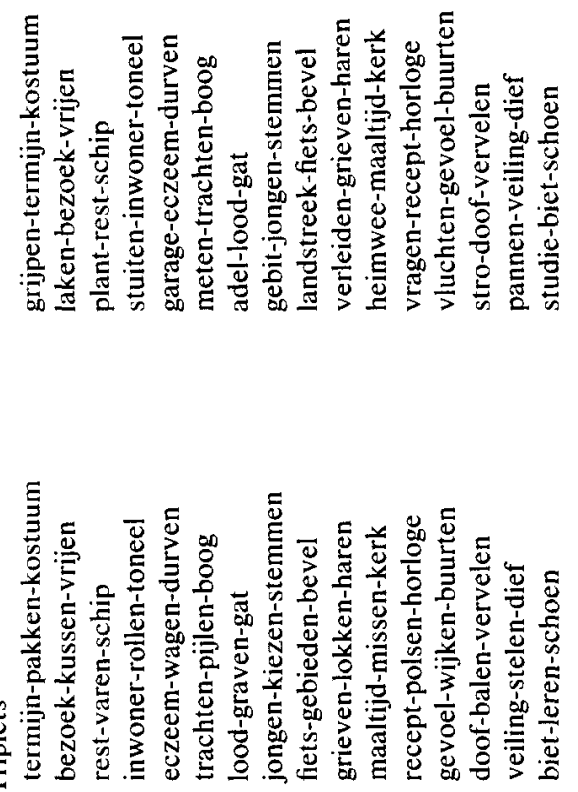

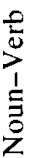
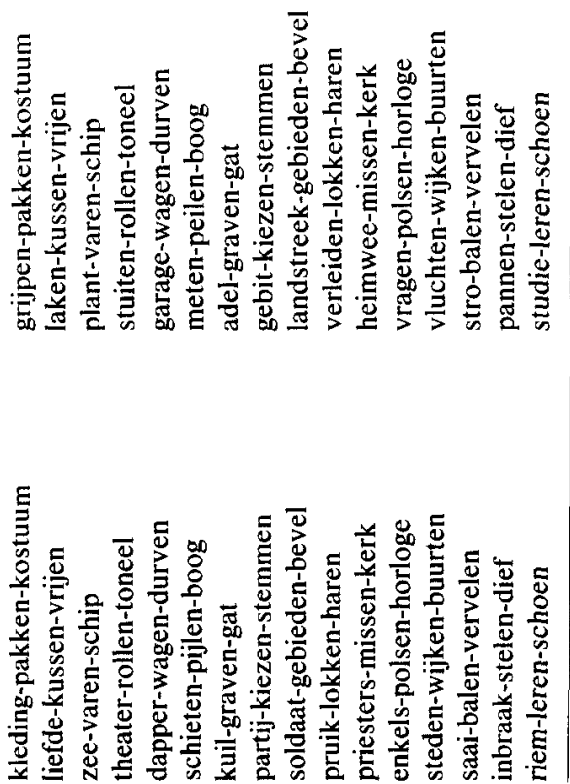


\section{REFERENCES}

Blumstein, S. E., Milberg, W., \& Shrier, R. 1982. Semantic processing in aphasia: Evidence from an auditory lexical decision task. Brain and Language, 17, 301-315.

Caramazza, A., \& Berndt, R. S. 1978. Semantic and syntactic processes in aphasia: A review of the literature. Psychological Bulletin, 85, 898-918.

Forster, K. I. 1979. Levels of processing and the structurc of the language processor. In W. E. Cooper \& E. C. T. Walker (Eds.), Sentence processing. Hillsdale, NJ: Erlbaum.

Milberg, W., \& Blumstein, S. E. 1981. Lexical decision and aphasia: Evidence for semantic processing. Brain and Language, 14, 371-385.

Milberg, W., Blumstein, S. E., \& Dworetzky, B. 1987. Processing of lexical ambiguities in aphasia. Brain and Language, 31, 138-150.

Scarborough, D. L., Cortese, C., \& Scarborough, H. S. 1977. Frequency and repetition effects in lexical memory. Journal of Experimental Psychology: Human Perception and Performance, 3, 1-17.

Schvaneveldt, R. W., \& Meyer, D. E. 1973. Retrieval and comparison processes in semantic memory. In S. Kornblum (Ed.), Attention and Performance IV. New York: Academic Press.

Schvaneveldt, R. W., Meyer, D. E., \& Becker, C. A. 1976. Lexical ambiguity, semantic context, and visual word recognition. Journal of Experimental Psychology: Human Perception and Performance, 2, 243-256.

Seidenberg, M. S., Tanenhaus, M. K., Leiman, J. M., \& Bienkowski, M. 1982. Automatic access of the meanings of ambiguous words in context: Some limitations of knowledgebased processing. Cognitive Psychology, 14, 489-537.

Swinney, D. A. 1979. Lexical access during sentence comprehension: (Re)consideration of context effects. Journal of Verbal Learning and Verbal Memory, 18, 645-659.

Winer, B. J. 1971. Statistical principles in experimental design. New York: McGraw-Hill. 